\title{
Surgical Resection of Malignancies Invading Inferior Vena Cava Level I and II. Issues Still Need to Be Discussed
}

\author{
EMILIO VICENTE, YOLANDA QUIJANO, BENEDETTO IELPO, HIPOLITO DURAN, EDUARDO DIAZ, \\ ISABEL FABRA, LUIS MALAVÉ, VALENTINA FERRI, ANTONIO FERRONETTI and RICCARDO CARUSO
}

Sanchinarro University Hospital, Madrid, Spain

\begin{abstract}
Background/Aim: Abdominal malignancies invading inferior vena cava present high postoperative morbidity and poor prognosis that has been decreasing in the last years. Our aim was to present and discuss a series of resected tumors invading the inferior vena cava from the origin of the hepatic vein to the common iliac veins (Level I and II). Materials and Methods: We retrospectively evaluated from 2005 to 2015 short- and long-term results of 20 consecutive surgical resections of tumors with associated inferior vena cava resection at Levels I and II performed at the Sanchinarro Hospital, Madrid, Spain. Results: The series included 4 leiomyosarcomas, 3 pancreatic cancers, 2 germinal cancers, 5 renal cancers, 4 liver cancers and 2 adrenal cancers. There were 7 circular cava resections, all of them replaced by polytetrafluoroethylene (PTFE) graft and 13 primary repairs. Sapheno-femoral arterio-venous fistulas have been performed in 6 cases. Early postoperative complications occurred in 11 cases (57.9\%) and mortality in one case. Graft thrombosis occurred in one case at 14 months from surgery $(1 / 7,14.2 \%)$. Conclusion: Surgical resection with combined inferior vena cava resection can be performed in selected patients with acceptable morbidity and mortality in light of the recent advancement in surgical technique.
\end{abstract}

Abdominal tumors involving the inferior vena cava (IVC) can originate from the vein itself (leiomyosarcoma) or from malignant tumors of nearby tissues (kidney, liver, pancreas, duodenum, geminal line tumors, etc.) (1). There are 2 types of classifications that divide the IVC in different levels by anatomy (Kulayat and Neves-Zincke type) $(1,2)$. The Neves-Zincke type is mainly used to describe the extension of the tumoral thrombi from renal cancer; however, on a surgical point of view, it is more accurate for planning a surgical resection of tumors

Correspondence to: Benedetto Ielpo, Sanchinarro University Hospital, Calle Oña 10, 28050, Madrid, Spain. Tel: +34 917567800, Fax: +34 917500133, e-mail: ielpo.b@gmail.com

Key Words: Inferior vena cava, graft thrombosis, cavotomy. affecting the IVC. This classification divides IVC in: Level I, lower level (IVC below renal veins); Level II, middle level (renal veins to hepatic veins); and Level III, upper level (entry of hepatic veins to right atrium); level IV when the IVC involvement includes also the right atrium. Resection of tumors involving level I and II are the most frequent.

Surgery is considered the best option of care and provides the only cure possibility for these types of tumors. In light of recent oncological and diagnostic advancement, an increasing number of cases undergo surgical resection, still with significant morbidity and mortality. It is important to note that only few studies address some technical and clinical issues that need to be further discussed (3-5).

Thus, the aim of this study was to show our recent experience in resection of tumors invading the IVC from the origin of the hepatic veins to the common iliac veins (Level I and II) (2) and discuss some technical issues.

\section{Materials and Methods}

Data collection. During the past 10 years (from January 2005 to January 2015), a total of 35 patients underwent surgical resection for malignant tumors involving the IVC at our center. Among them, only patients with infrarenal and infra hepatic cava vein involvement were selected to be retrospectively analyzed (Level I and II) (2). Remaining patients had tumor involving Level III and $\mathrm{IV}$, therefore, excluded from this analysis. Preoperative variables examined include age, sex, body mass index (BMI), American Society of Anesthesiologist classification (ASA), tumor type, preoperatory stage and concomitant organ involvement, preoperative chemotherapy and radiotherapy. Perioperative clinical results include operative time, blood transfusion, surgical approach, type of IVC resection and reconstruction. Main short-term data recorded include length of hospital stay, complication rate (according to the Clavien classification; severe complication is defined as greater or equal to IIIb) (6), re-operation, re-admission and early postoperative mortality $(<60$ days after operation). Operative mortality was defined as death during the hospital stay or within 90 days from surgery. Main long-term data recorded include late postoperative mortality ( $>90$ days after operation), morbidity related to surgery, femoral arterio-venous fistula (FAV) patency, specific disease-free survival (DSF) and overall survival (OS). OS for patients who underwent surgery was calculated starting from the diagnosis. 
Preoperative management. Clinical examination, blood analysis and electrocardiogram were routinely performed in all patients. Computed tomography (CT) scan of the abdomen and the chest with intravenous contrast was obtained in order to identify tumor extension, infiltration to surrounding structures and resectability, as well as sites of metastasis. CT urography renal scintigraphy was also assessed when required. Abdominal magnetic resonance imaging (MRI) and positron emission tomography (PET) CT scan were additionally used for diagnosis. When carcinomatosis was suspected, abdominal explorative laparoscopy was performed with concomitant ecolaparoscopy in order to better assess resectability. According to the histologic type, patients were evaluated by a multidisciplinary team and specific neoadjuvant treatments were administered.

Surgeons. The procedures were performed by the two senior surgeons with high hepato-pancreato-biliary surgery experience, with the assistance of 6 more surgeons and trainees.

Surgical approach. If there is a circular IVC resection, prosthesis of polytetrafluroethylene (PTFE) of $20 \mathrm{~mm}$ of diameter is used to substitute it. When long prosthesis is required $(>6-7 \mathrm{~cm})$, a concomitant left sapheno-femoral arterio-venous fistula is usually performed, in order to assure an adequate IVC flow, decreasing the risk of thrombosis (Figure 1A and B). If only a tangential resection is needed, whenever it is feasible, a primary longitudinal or transversal suture is performed with 4-0 prolene.

Heparin is not used during the clamping time of the IVC, and only a prophylactic heparin dose is used during the short postoperative period of time (almost 30 days after surgery).

When needed, a FAV was performed bewteen the saphenal vein and the femoral artery.

Retroperitoneal lymphadenectomy. We performed retroperitoneal lymphadenectomy for adrenal, kidney and leiomyosarcomas tumors.

Follow-up. Patients underwent multidisciplinary (oncologists and surgeons) revisions at almost every 5-6 months with measurement of tumoral markers, chest-abdominal CT. Local recurrence was defined as recurrent tumor within the surgical field, whereas systemic recurrence was defined as recurrent disease outside.

\section{Results}

A total of 20 surgical resections of tumors involving IVC Level I and II have been performed and recorded. There were 12 males and 8 females with a mean age of 53.2 years (range $=22-70$ years). Tumors types are showed in Table I including 10 tumors of Level I, 8 of Level II and 2 tumors extended to both Level I and II. Mean follow-up was 42.7 months (range $=19-90$ months). No patient was lost during follow-up. Only patients undergoing surgery for pancreatic adenocarcinoma, germinal tumors and colorectal liver metastases (CRLM) received neoadjuvant treatment (Table I).

Type of surgery. Radical resection of leiomyosarcoma with circular IVC resection, replaced by a $20-\mathrm{mm}$ diameter PTFE graft, was performed in all 4 cases (Figure 2A, B; Table I). In 2 cases, a right nephrectomy was also needed in which left renal veins have not been reconstructed.

Pancreatectomies with partial involvement of IVC have been performed in 3 cases (1 duodeno-chephalo pancreatectomy, 1 distal pancreatectomy, 1 total pancreatectomy). In all of these cases, only a partial IVC resection was performed and, therefore, sutured transversely with prolene 4/0. Two radical resections of germinal line tumors have been included. One case with partial IVC resection and the other with complete circular IVC resection were replaced by PTFE graft.

Radical nephrectomy was performed in 5 cases with one case of complete IVC resection and PTFE replacement after left renal nephrectomy. After graft placement, the right venal rein was reconnected in the IVC above the graft.

A total of 4 liver resections were also included (2 CRLM and 2 hepatocellular carcinomas (HCC), including 1 case where the IVC was replaced by PTFE graft.

Two cases of adrenalectomy were performed with partial IVC resection repaired with direct suture.

The length of PTFE graft used for each type of procedure is shown in Table 2. Main postoperative data for each malignancy are shown in Table II. Blood transfusion was required in 16 cases $(80 \%)$.

$F A V$. Sapheno-femoral arterio-venous fistula has been performed in 6 cases, except in one case of a CRLM patient as the lenght of the graft used was short $(5 \mathrm{~cm})$.

Postoperative short-term data. Early postoperative complications occurred in 11 cases $(57.9 \%)$. They included Clavien-Dindo grades I $(n=0)$, II $(n=3)$, IIIa $(n=4)$, IIIb $(\mathrm{n}=2), \mathrm{IVb}(\mathrm{n}=1)$ and $\mathrm{V}(\mathrm{n}=1)$. The only grade $\mathrm{V}$ case $(5.2 \%$ of all series) occurred after distal pancreatectomy at the $32 \mathrm{nd}$ postoperative day. This patient died after a multi-organ failure syndrome secondary to pancreatic fistula. Only 4 (21\%) of complications were classified as severe. Mean length of hospital stay was 21 days (range=9-85).

When indicated, postoperative adjuvant treatment was administered as shown in Table II.

Early postoperative outcomes. Mean OS and mean DFS of this series was 42 and 35.6 months, respectively. Diseasespecific OS and DFS are shown in Table II. A case of leiomyosarcoma developed retroperitoneal recurrent disease with partial IVC stenosis at the PTFE site after 36 months from the operation. This patient is under investigation in order to plan new surgery.

Thrombosis of PTFE graft occurred in one case (1/7: $14.2 \%)$ after 14 months from the operation. It was managed conservatively as collateral venous system was established without any clinical consequence. 


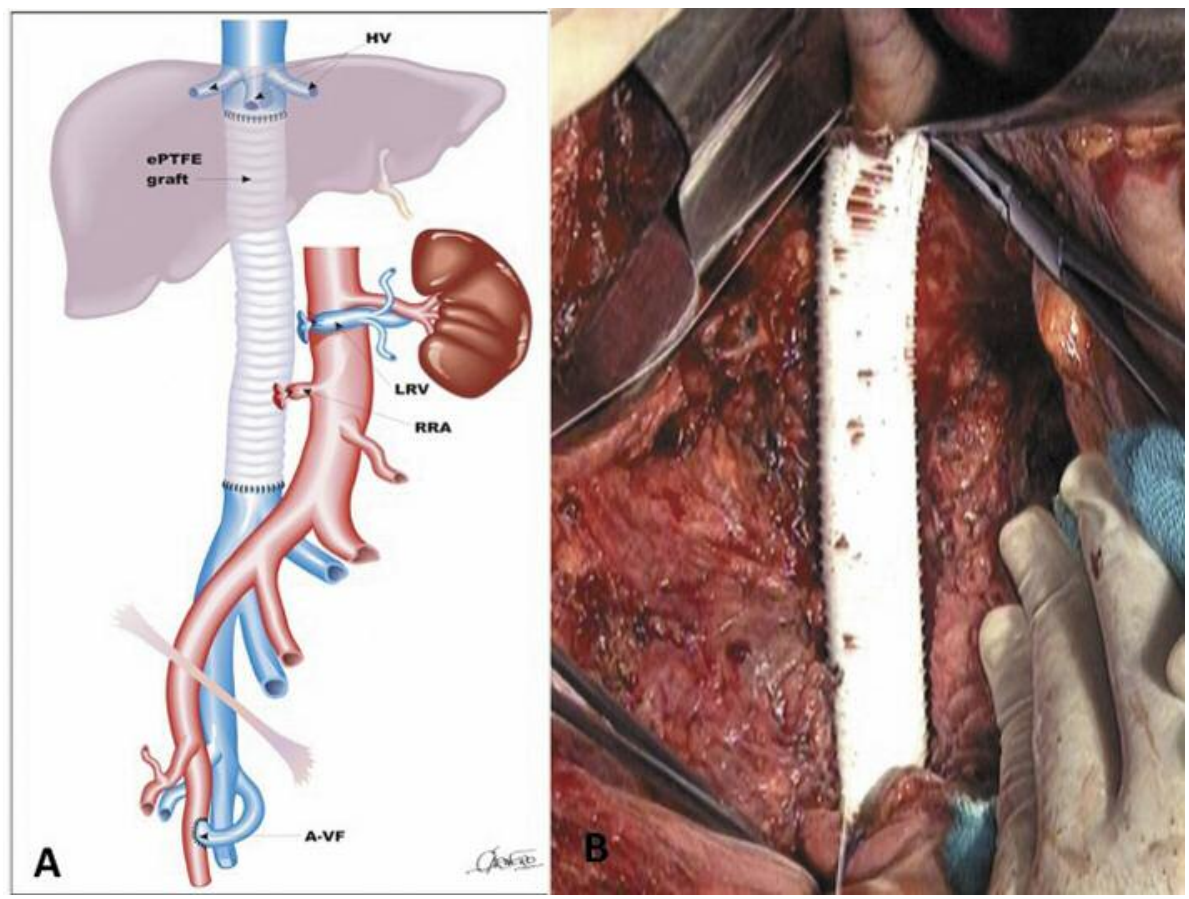

Figure 1. A: Polytetrafluroethylene (PTFE) graft with femoral arterio-venous fistula; B: Large PTFE graft.

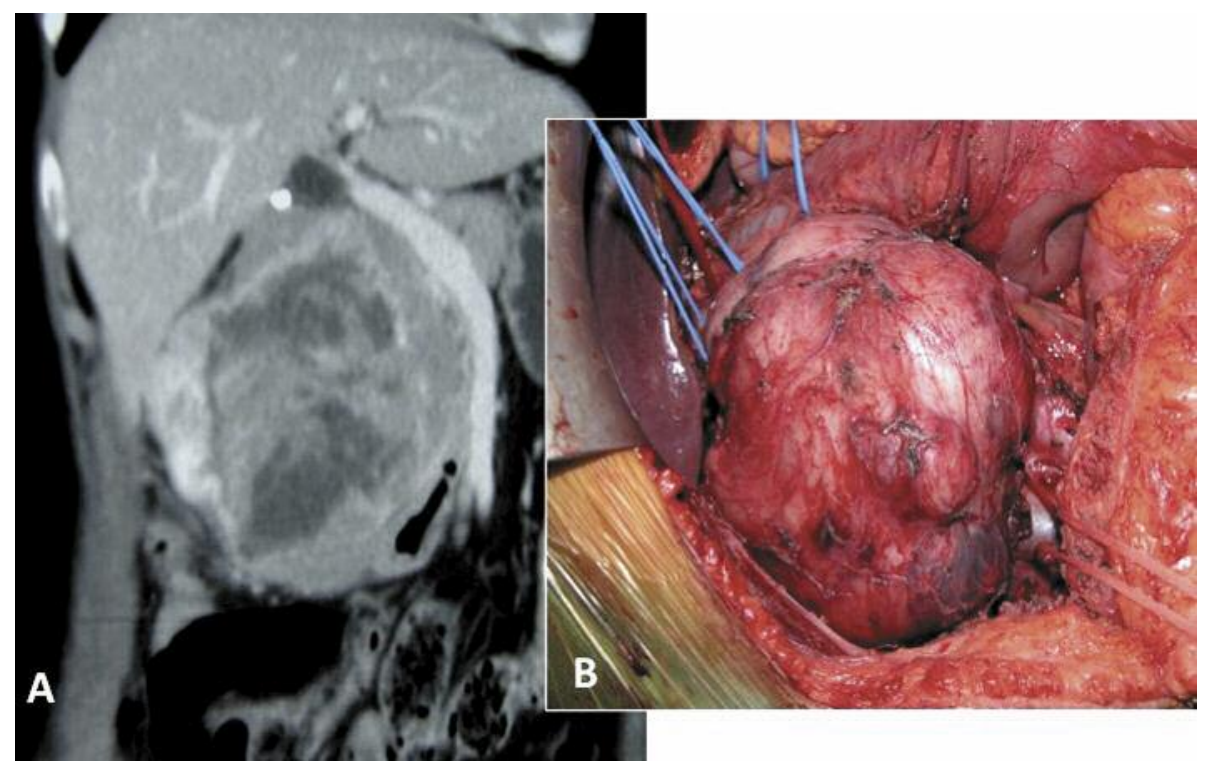

Figure 2. A: Giant leiomyosarcoma at computed tomography (CT) scan; B: Intraoperative finding of giant leiomyosarcoma.

\section{Discussion}

Surgical resection for patients with locally advanced malignancy, involving IVC, remains the only hope for cure or palliative care for their symptoms. This procedure is rarely performed because of the magnitude of the risk of the operation and because most of these patients are at advanced stages with distant metastases (3). 
Table I. Main patients' characteristics.

\begin{tabular}{|c|c|c|c|c|c|c|c|c|c|c|}
\hline Type of tumour & $\begin{array}{l}\text { No. of } \\
\text { patients }\end{array}$ & $\begin{array}{l}\text { Gender } \\
\text { (range) }\end{array}$ & $\begin{array}{l}\text { Mean age } \\
\text { treatment }\end{array}$ & $\begin{array}{l}\text { ASA } \\
\text { level }\end{array}$ & $\begin{array}{l}\text { Neoadjuvant } \\
\text { intervention }\end{array}$ & $\begin{array}{c}\text { IVC } \\
\text { treatment }\end{array}$ & $\begin{array}{c}\text { Type of surgical } \\
\text { (number; Type; } \\
\text { lenght) }\end{array}$ & Adjuvant & Prosthesis & FAV \\
\hline Leiomyosarcomas IVC & 4 & $3 \mathrm{~F}$ & $58,3(45-69)$ & $4 \mathrm{II}$ & none & 2 I; 2 II & 3 circular & Radiotherapy & $\begin{array}{c}4, \text { PTFE; } \\
(5,7,9 \\
13 \mathrm{~cm})\end{array}$ & 3 \\
\hline Pancreatic cancer & 3 & $2 \mathrm{M} ; 1 \mathrm{~F}$ & $52,6(48-56)$ & 2 II, $1 \mathrm{I}$ & $\begin{array}{l}\text { Nab paclitaxel, } \\
\text { Gemcitabine } \\
\text { Radiotherapy }\end{array}$ & II & 3 partial & Gemcitabine & NO & $\mathrm{NO}$ \\
\hline Germ tumors & 2 & $2 \mathrm{M}$ & $23(22-24)$ & 2 II & $\mathrm{BEP}$ & I & $\begin{array}{l}1 \text { circular } \\
1 \text { partial }\end{array}$ & BEP & $\begin{array}{c}\text { 1, PTFE, } \\
7 \mathrm{~cm}\end{array}$ & 1 \\
\hline Renal tumors & 5 & $3 \mathrm{M} ; 2 \mathrm{~F}$ & $55(26-71)$ & $2 \mathrm{II}$ & none & $3 \mathrm{I} ; 2 \mathrm{II}$ & $\begin{array}{l}1 \text { circular } \\
4 \text { partial } \\
1 \mathrm{RRV} \\
\text { reinsertion }\end{array}$ & none & $\begin{array}{c}\text { 1, PTFE, } \\
20 \mathrm{~cm}\end{array}$ & 1 \\
\hline $\begin{array}{l}\text { Hepatic tumors } \\
\text { 2CRLM } \\
2 \mathrm{HCC}\end{array}$ & 4 & $2 \mathrm{M} ; 2 \mathrm{~F}$ & $55,7(43-63)$ & 2II, 2III & $\begin{array}{l}\text { 5FU, Xelox, } \\
\text { Avastin }\end{array}$ & II & $\begin{array}{l}1 \text { circular } \\
3 \text { partial }\end{array}$ & $\begin{array}{c}\text { 5FU, Xelox } \\
\text { Avastin } \\
\text { Sorafenib }\end{array}$ & $\begin{array}{c}\text { 1, PTFE } \\
5 \mathrm{~cm}\end{array}$ & $\mathrm{NO}$ \\
\hline Adrenal tumours & 2 & $2 \mathrm{M}$ & 70 & & none & II & 2 partial & none & No & NO \\
\hline
\end{tabular}

M, Male; F, female; HCC, hepatocellular carcinoma; CRLM, colorectal liver metastases; IVC, inferior vena cava; FAV, femoral arterio-venous fistula; PTFE, polytetrafluroethylene; BEP, bleomycin, etoposid, cisplatin; 5FU, 5-fluorouracil; RRV, right renal vein.

Table II. Main post-operative outcome.

\begin{tabular}{lcccccc}
\hline Type of tumor & $\begin{array}{c}\text { Patients with } \\
\text { transfusions } \\
\text { (mean, ml) }\end{array}$ & $\begin{array}{c}\text { Clavien-Dindo } \\
\text { score } \\
\text { type }\end{array}$ & $\begin{array}{c}\text { Graft } \\
\text { complications }\end{array}$ & $\begin{array}{c}\text { Mean hospital } \\
\text { stay (range) } \\
\text { days }\end{array}$ & $\begin{array}{c}\text { Mean overall } \\
\text { survival (range) } \\
\text { months }\end{array}$ & $\begin{array}{c}\text { Mean disease } \\
\text { free survival } \\
\text { (range) months }\end{array}$ \\
\hline Leiomyosarcomas IVC & 3,120 & 1 II; 1 IIIb & None & $17.3(13-20)$ & $55(29-60)$ & $30(25-50)$ \\
Pancreatic cancer & 2,100 & 1 IVb; 1 V & - & $37(15-64)$ & $41(21-58)$ & $38.6(14-58)$ \\
Germ tumors & 2,130 & 1 II & None & $10(9-11)$ & $46(34-58)$ & $44.5(33-56)$ \\
Renal tumors & 5,160 & 3 IIIa & None & $27.4(12-85)$ & $50.6(26-90)$ & $44.4(20-90)$ \\
Hepatic tumors & 4,110 & 1 II ; 1 IIIa; 1 IIIb & 1 late thrombosis * & $13(11-14)$ & $36(29-48)$ & $29.5(18-48)$ \\
Adrenal tumors & 1,60 & - & - & 17 & 19 & 14 \\
\hline
\end{tabular}

IVC, Inferior vena cava; $* 14$ months after operation of colorectal liver metastases.

However, as reported in the literature $(3,5,7)$, in the last decade, the number of procedures performed seems to be increasing, thus entailing the need for further discussion of some topics:

It has been hypothesized that slow tumor growth allows for development of venous collaterals, allowing for safe ligation of the IVC. However, the resection of large retroperitoneal tumors, as well as the retroperitoneal lymphadenectomy (performed when required), may disrupt the venous collaterals with a significant lower extremity edema in up to $50 \%$ of patients when the IVC is ligated. For this reason, it is suggested, whenever possible, to maintain the continuity of IVC by direct suture or replacing it with a prosthesis $(9,11)$. IVC ligation may be considered in some exceptional cases where the inflammatory reaction of the surgical field can be too dangerous for suturing or grafting and only if retroperitoneal lymphadenectomy is not required, like the case of a liver hydatid cyst involving IVC.

Currently, multiple materials for reconstruction are available, including autologous materials, such as saphenous vein graft or peritoneum, allografts, such as aortic homografts, xenografts, such as bovine pericardium. However, in the last decade, graft synthetic mesh reconstruction (including Dacron and PTFE) have become a progressively better and more available option. They have showed only a $2 \%$ graft-related complication rate with an excellent patency $(8,9)$. For this reason, their use has increased lately (8-10). 
For replacement of the IVC, in our experience, we used exclusively the ringed $20-\mathrm{mm}$ diameter PTFE graft. Some authors report that this graft is preferable than Dacron based on long-term patency.

There are series reporting that a smaller graft $(12-14 \mathrm{~mm})$ is preferable because it promotes faster flow velocities throughout the graft segment and avoids graft thrombosis (9, 10). However, the relatively low number of patients and the short follow-up of these studies cannot deduce strong conclusions on this issue.

We only used grafts with a diameter of $20 \mathrm{~mm}$ because the diameter of the IVC usually extends to over $15 \mathrm{~mm}$ and the use of a smaller PTFE graft may tend to form a thick pseudointima or even thrombus. According to our experience reported herein, the rate of PTFE graft thrombosis is $14.2 \%$ ( 1 out 7 cases) over a mean follow-up of 31.6 months. The thrombosis occurred in a late postoperative period (14 months) and it was the case where FAV was not performed because the length of the graft was considered to be short $(5 \mathrm{~cm})$.

A duodenocaval fistula has been recently reported in a patient with resection of IVC for leiomyosarcoma replaced by a PTFE graft (11). This underreported complication must be further investigated in future studies.

Indication to perform a FAV to decrease prosthesis thrombi is still under debate. Some authors insist that a FAV is not required if the suprarenal or suprahepatic vena cava is reconstructed because of the large blood flow volume at this level (10). However, we believe that this fistula may enhance graft patency for long $(>6-7 \mathrm{~cm})$ and shorter grafts. As mentioned before, in a CRLM case, even if a FAV was not indicated as the graft length was short, the patient developed a late graft thrombus. Therefore, our suggestion is to perform a FAV whenever there is need for prosthesis. Giving the retrospective nature and number of cases of this study, our data are insufficient to define the role of FAV in this clinical setting, considering also the new available anticoagulant treatments.

Some authors describe long-term complications secondary to FAV, such as congestive heart failure or edema. However, these complications are infrequent and usually occur only after a long period of time (12). In fact, according to our experience, there was not a need for a FAV closure to manage long-term complications in any patient. More experience is required to collect more data.

It has been shown that the left renal vein can be resected without reconstruction and with no need to nephrectomy. This transaction, however, must be proximal to the ascending lumbar vein and adrenal vein.

In the present study, 2 patients underwent left renal vein closure and concomitant right nephrectomy; postoperative renal function was preserved in all cases. Therefore, according to our experience and literature review, it is a safe procedure (9) and should be taken into consideration whenever needed.
The use of heparin during the clamping time of the IVC and in the postoperative period is still under discussion since there are no studies addressing this topic in the literature. In our normal practice, heparin is not used during the clamping time of the IVC and only a prophylactic heparin dose is used up to almost 30 days from surgery. The real benefit of the use of some anticoagulant treatment after IVC resection needs to be better evaluated in larger, comparative series (13).

Novel chemotherapeutic drugs have been introduced recently for these types of malignancies. New adjuvant treatments allow a better selection of patients that will benefit from this aggressive surgery. According to our experience, a total of 9 patients underwent surgery after neoadjuvant treatment (Table I). In this setting, surgery should be planned only if patients have a good response to this treatment. Multiagent chemotherapy represents an important tool to achieve a potentially $\mathrm{R} 0$ resection, especially for locally advanced pancreatic adenocarcinoma with IVC involvement, as shown in our 3 cases. Furthermore, it allows also selecting those patients who are really going to benefit from this type of aggressive surgical resection.

Actually, our routine work-up consists in giving pancreatic adenocarcinoma patients a chemoradiotherapy regimen before surgery (gemcitabine plus nab-paclitaxel), as shown in Table I. Only patients with good response to neoadjuvancy should undergo surgery.

A new targeted drug regimen includes sorafenib, which, in our series, was given to 2 cases of HCC. This drug has been shown to maximize overall survival and disease progression control in unresectable and resectable HCC (14).

The use of radiotherapy after leiomyosarcoma resection is under debate. We agree with authors suggesting its use only if there is a margin involvement after resection (7). In our series none of the patients underwent adjuvant radiotherapy as all margins were free from disease.

Careful patient selection is critical to achieve better outcomes. Recent improvements of the imaging techniques allow a better selection of patients going to benefit from this aggressive approach. First, they allow better identification of underling distal metastases that may contraindicate surgery, as it is shown by PET CT scan and PET MRI. Second, they provide adequate information about tumor invasion, associated thrombi, vascular anomalies, as shown by abdominal CT scan with vascular and 3-dimension reconstruction. These techniques are paramount to plan the right surgical strategy for these types of aggressive diseases minimizing worthless operation. Currently, laparoscopic exploration with echolaparoscopy previous to definitive surgery has an important role on decision-making.

The main claimed benefit of neoadjuvant therapy is to improve OS. OS depends primary on R0 resection and, therefore, on an adequate selection of patients suitable for surgery. This is the scenario where preoperative treatment can 
make the difference as neoadjuvant therapy may only identify patients with particularly aggressive disease (non-responders) who would probably not benefit from resection. This is especially important in the case of locally advanced pancreatic cancers, due to the higher rate of postoperative complications among them. The 3 reported cases of locally advanced pancreatic cancer with IVC involvement had a good response to preoperative treatment and, therefore, selected for this aggressive surgery, showing a high OS and DFS (Table II).

A multidisciplinary approach with oncologists, radiologists, pathologists and surgeons is important to further define a better treatment strategy for these patients.

\section{Conclusion}

According to our experience, resection of tumor associated with IVC, involving Level I and II, is associated with an acceptable postoperative morbidity and mortality. Some topics of this procedure need to be further investigated.

\section{Conflicts of Interest}

None.

\section{Acknowledgements}

The Authors thank Jan Lammel, Pablo Ruiz and Isabel de Salas for their contribution.

\section{References}

1 Eder F, Halloul Z, Meyer F, Huth C and Lippert H: Surgery of inferior vena cava associated malignant tumor lesions. Vasa 37(1): 68-80, 2008.

2 Neves RJ and Zincke H: Surgical treatment of renal cancer with vena cava extension. Br J Urol 59(5): 390-395, 1987.

3 Haddad AQ, Wood CG, Abel EJ, Krabbe LM, Darwish OM, Thompson RH, Heckman JE, Merril MM, Gayed BA, Sagalowsky AI, Boorjan SA, Margulis V and Leibovich BC: Oncologic outcomes following surgical resection of renal cell carcinoma with inferior vena caval thrombus extending above the hepatic veins: A contemporary multicenter cohort. J Urol 192(4): 1050-1056, 2014.

4 Parissis H, Akbar MT, Tolan M and Young V: Surgical resection of a renal cell carcinoma involving the inferior vena cava: The role of the cardiothoracic surgeon. J Cardiothorac Surg 5(5): 103, 2010.
5 Alejo JL, George TJ, Beaty CA, Allaf ME, Black JH 3rd and Shah AS: Novel approach to recurrent cavoatrial renal cell carcinoma. Ann Thorac Surg 93(5): e119-121, 2012.

6 Dindo D, Demartines N and Clavien PA: Classification of surgical complications: A new proposal with evaluation in a cohort of 6,336 patients and results of a survey. Ann Surg 240(2): 205-213, 2004.

7 Nigri G, Petrucciani N, Sirimarco D, Mangogna L, Aurello P and D'Angelo F: Ramacciato G. Inferior vena cava resection without prosthesis replacement for vena cava sarcoma (with video). J Visc Surg 153(5): 387-388, 2016.

8 Alexander A, Rehders A, Raffel A, Poremba C, Knoefel WT and Eisenberger CF: Leiomyosarcoma of the inferior vena cava: radical surgery and vascular reconstruction. World J Surg Oncol 7: 56, 2009.

9 Wang Q, Jiang J, Wang C, Lian G, Jin MS and Cao X: Leiomyosarcoma of the inferior vena cava level II involvement: Curative resection and reconstruction of renal veins. World $\mathrm{J}$ Surg Oncol 10: 20, 2012.

10 Armstrong PA, Back MR, Murray L, Shames ML, Bailey CJ, Kim T, Lawindy SM, Sexton WJ and Spiess PE: Outcomes after inferior vena cava thrombectomy and reconstruction for advanced renal cell carcinoma with tumor thrombus. J Vasc Surg 2(4): 368-376, 2014.

11 Ippolito D, Querques G, Drago SG, Bonaffini PA and Sironi S: Duodenocaval fistula in a patient with inferior vena cava leiomyosarcoma treated by surgical resection and caval polytetrafluoroethylene prosthesis. Case Rep Radiol 2015: 575961, 2015.

12 Wachtel H, Jackson BM, Bartlett EK, Karakousis GC, Roses RE, Bavaria JE and Fraker DL: Resection of primary leiomyosarcoma of the inferior vena cava (IVC) with reconstruction: A case series and review of the literature, J Surg Oncol 111(3): 328-333, 2015.

13 Spinelli F, Stilo F, La Spada M, De Caridi G and Benedetto F: Surgical treatment of tumors involving the inferior vena cava. Personal experience. J Cardiovasc Surg 49(3): 323-328, 2008.

14 Kudo M: Recent trends in the management of hepatocellular carcinoma with special emphasis on treatment with regorafenib and immune checkpoint inhibitors. Dig Dis 34(6): 714-730, 2016. 